\title{
Kaizen: Filosofía de mejora continua El caso Facusa
}

\author{
Jorge López Saldarriaga \\ Facusa SAC \\ Ingeniería Industrial n. 28, 2010, ISSN 1025-9929, pp 41-57 \\ Recibido: 2 de junio del 2010 / Aprobado: 7 de julio del 2010
}

\begin{abstract}
RESUMEN: El objetivo primordial de este artículo es motivar a todas aquellas organizaciones que buscan asumir los retos que les impone la apertura de los mercados internacionales y los tratados de libre comercio, y tratan incansablemente de exceder las expectativas de sus clientes, tanto externos como internos, y finalmente de todos los grupos de interés. Mediante la aplicación de las herramientas de gestión, como las implementadas en Facusa bajo el marco de un proceso de mejora continua, las empresas pueden experimentar las infinitas posibilidades a las que tienen acceso.
\end{abstract}

Palabras clave: mejora continua / innovación / eficiencia / productividad

\section{Kaizen: Philosophy of continuous improvement Case Facusa}

ABSTRACT: The primary objective of this article is to encourage all organizations seeking to meet the challenges derived from the opening of international markets and free trade agreements, and trying tirelessly to exceed the expectations of both external and internal customers and, ultimately, of all interest groups. Through the application of management tools, like the one implemented at Facusa under the framework of a continuous improvement process, organizations can experience the endless possibilities they can access.

Keywords: continuous improvement / innovation / efficiency / productivity 


\section{INTRODUCCIÓN}

Kaizen es una palabra japonesa que encierra la filosofía de la mejora continua enfocada a todos los aspectos de nuestra vida; sin embargo, en esta oportunidad trataremos sobre el impacto que este enfoque de mejora continua ha tenido en la empresa peruana de cubiertos Facusa.

Esta empresa peruana, que inició sus operaciones en la década de 1960, se vio enfrentada, a finales de la década de 1990, a los retos de la globalización y la apertura del mercado peruano, y tuvo que competir con productos de precios muy atractivos procedentes de diversas partes del mundo.

\section{DIAGNÓSTICO DE LA EMPRESA}

Antes de iniciar el proceso de cambio, Facusa se encontraba en una situación de desventaja para competir en un mercado globalizado. Entre los principales problemas que tenía la empresa estaban los siguientes:

- Procesos de producción intensivos en mano de obra.

- Tiempos de entrega largos.

- Poca flexibilidad de sus procesos.

- Tiempos elevados de preparación de máquina.

- Poca variedad de productos.

- Elevados niveles de reproceso.

- Elevado nivel de producto no conforme.

- Elevados niveles de desperdicio (Muda). ${ }^{1}$

- Puestos de trabajo sucios y desordenados.

- Productos con poco y ningún margen de contribución.

- Ventas dirigidas únicamente al mercado local.

- Ausencia de definición de objetivos y metas.

- Procesos administrativos lentos.

- Poco acercamiento con los proveedores.

1 Término tradicional japonés que nombra cualquier actividad que significa desperdicio o que no agrega valor. 
- Personal poco motivado y capacitado.

- Poco o nulo desarrollo tecnológico.

- Decisiones tardías, poca información.

- Bajo nivel de innovación.

- Bajo nivel de compromiso de los trabajadores.

- Reclamaciones de clientes.

- Constante rotura de stock de producto terminado.

Esta complicada situación tenía que cambiar lo antes posible si la empresa deseaba permanecer en el mercado.

El cambio se inició en 1999, con el desarrollo de un planeamiento estratégico que evidenció las fortalezas, oportunidades, debilidades y amenazas a las que se enfrentaba nuestra empresa si continuábamos trabajando bajo el mismo esquema que había tenido relativo éxito en el pasado. Este análisis nos obligó a redefinir nuestra estrategia, en realidad para ser más fiel a lo que sucedió, nos confrontó dramáticamente con la realidad de competir en un mercado globalizado y los retos que esto implicaba.

El cambio se inició al reconocer quiénes éramos y en qué queríamos llegar a convertirnos, identificando en este proceso nuestros valores empresariales. Posteriormente, luego de definir nuestro enfoque de trabajo basado en el cliente planteamos nuestros objetivos y metas, así como las estrategias e iniciativas de mejora que nos permitirían concretar estos objetivos empresariales.

La misión, la visión y los valores corporativos que la alta dirección de Facusa definió al inicio de este proceso de mejoramiento continuo y que han orientado permanentemente nuestros esfuerzos durante la última década fueron los siguientes:

- Misión.- "Somos una empresa peruana dedicada a producir cubiertos de mesa, cuchillos y utensilios de cocina en acero inoxidable de alta calidad, que procura atender todas las necesidades de nuestros clientes, mejorando permanentemente nuestros procesos y productos".

- Visión.- "Consolidarnos durante la presente década como líderes sudamericanos en la fabricación de cubiertos de mesa, cuchillos y utensilios de cocina en acero inoxidable de la más alta calidad, para el mercado nacional e internacional utilizando tecnología automa- 
tizada de punta y fomentando el desarrollo de las actividades productivas dentro de un marco de mejora continua, buscando exceder las expectativas de nuestros clientes y contribuyendo con la generación de fuentes de trabajo".

- Valores corporativos.-

- Eficacia: Cumplimos en los plazos previstos las metas trazadas.

- Servicio: El cliente es la razón de ser de nuestro negocio.

- Respeto: Considerar a todo el personal de nuestra organización sin hacer distinción alguna.

- Innovación: La calidad en todas nuestras acciones, orientada siempre a la mejora continua.

- Trabajo en equipo: Unión de esfuerzos de los integrantes de la organización orientados a alcanzar objetivos y metas comunes.

- Eficiencia: Utilizamos nuestros recursos racionalmente.

Entre los principales desafíos que enfrentó Facusa durante la última década, y que estuvieron relacionados con el cumplimiento de su misión y la puesta en marcha de acciones estratégicas para alcanzar su visión, podemos mencionar:

- Liderazgo en costos en sus líneas de cuchillos y utensilios de cocina a través de la mejora continua de productos y procesos, la introducción de tecnología de punta y la reducción agresiva del desperdicio.

- Diferenciación en sus líneas de cubiertos de mesa a través de una estrategia de innovación, desarrollo tecnológico e ingeniería del valor.

- Penetración y posicionamiento en nuevos mercados sudamericanos por medio de la orientación a la calidad, la satisfacción del cliente y alianzas estratégicas con los intermediarios.

Por ello, la alta dirección de Facusa determinó que debía concentrar todos sus esfuerzos en la reducción de sus costos de producción, en mejorar la flexibilidad de sus procesos productivos, reducir los tiempos de fabricación y los tiempos de entrega, aumentar la rentabilidad de sus productos, mejorar el cumplimiento de los programas de producción, y su nivel de innovación tecnológica. Por otra parte, la empresa debía desarrollar ventajas competitivas relacionadas con el nivel de satisfacción de sus clientes, velocidad de respuesta, enfoque 
de segmentación de mercados y el desarrollo de relaciones estratégicas de mutuo beneficio con sus socios proveedores.

Lo que motivó la definición de la misión, la visión y los valores corporativos en Facusa fue la necesidad de añadir valor al proceso de cambio de cultura organizacional, ya que al plantearlas se desprendieron nuevas oportunidades que nos obligaron a trazar en torno a ellas estrategias específicas, que se constituyeron en los fundamentos de determinadas acciones de mejora que fueron de mucha utilidad para alinear, dirigir y controlar los esfuerzos y recursos de la organización.

Adicionalmente, resultó un camino muy efectivo para lograr un mayor compromiso de todos sus colaboradores, así como para que la organización tuviera un rumbo de desarrollo más claro. Posteriormente estos conceptos se consolidarían en su política de la calidad. Un factor determinante para lograr incorporar una nueva cultura de trabajo en Facusa, basada en la mejora continua, ha sido sin duda alguna el compromiso tanto de la alta dirección como de todos sus colaboradores, quienes luego de un periodo intensivo de capacitación han sido los pilares sobre los que la empresa apoya todas sus iniciativas de mejora.

\section{CRONOLOGÍA DEL CAMBIO EN FACUSA}

\section{0}

Implementa el sistema de costeo basado en actividades (ABC), lo que le permite a la empresa, mediante una mejor asignación de sus costos indirectos de fabricación - alrededor del 30\% dentro de su estructura de costos-, establecer el precio de sus productos de manera más precisa y tomar decisiones estratégicas con referencia al diseño y desarrollo de nuevos productos, determinando la rentabilidad por producto, identificando los productos con mayor margen de contribución y aquellos en los que era necesario un rediseño tanto del producto como del proceso para seguir compitiendo, o, en su defecto, dejar de producirlo.

Inicia su programa de mejora continua (Kaizen) conformando equipos de mejora y círculos de calidad, capacitándolos con las principales herramientas de calidad como tormenta de ideas, diagrama causaefecto, diagrama de Pareto, entre otras, que permitieron identificar 
rápidamente importantes oportunidades de mejora, logrando una reducción del desperdicio (Muda) relacionado con exceso de movimientos, exceso de inventarios, defectos en los productos, reproceso y merma de entre el $14 \%$ y el $25 \%$. El objetivo principal de este programa era, como lo menciona Domínguez Machuca en relación con las dos estrategias básicas del sistema JIT: "Eliminar toda actividad innecesaria o fuente de despilfarro, por lo que intenta desarrollar el proceso de producción utilizando un mínimo de personal, materiales, espacio y tiempo. Fabricar lo que se necesite, en el momento en que se necesite y con la máxima calidad posible" (Domínguez et al. 1995: 202).

\section{1}

Incorpora los conceptos de la TOC a su planeamiento y control de la producción, lo que le permitió, mediante el enfoque sistémico de la tecnología de producción optimizada, "[...] identificar las restricciones de su sistema, explotar dichas restricciones al máximo, subordinar todo al proceso más restrictivo (tambor) y elevar las restricciones del sistema para luego de eliminar una restricción iniciar el proceso completo nuevamente" (Domínguez et al. 1995: 271).

\section{2}

Implementa su programa de mejora 'Cinco S' en sus tres plantas de producción, lo que le permite reducir el desperdicio de movimientos, elevar la disciplina y crear un ambiente de trabajo superior, mejorar la seguridad y disminuir el número de accidentes, logrando aprovechar mejor el espacio disponible, bajar el porcentaje de productos defectuosos, minimizar las averías, generar una mayor cooperación y trabajo en equipo y sentido de pertenencia entre sus colaboradores, reducir el nivel de existencias o inventarios, desarrollar un mayor conocimiento del puesto de trabajo, reduciendo el tiempo de cambio de herramientas y consecuentemente incrementando la productividad de los puestos de trabajo en alrededor del 15\%.

Facusa se concentra en desarrollar colaboradores bajo el concepto de polivalencia, mediante la rotación de puestos y la adquisición de nuevas habilidades, lo cual da como resultado mayor flexibilidad y 


\section{Gráfico 1}

\section{Reducción de costos de mano obra directa en el área de almacén} luego del primer trimestre de la implementación de las $5 \mathrm{~S}$ ‘S

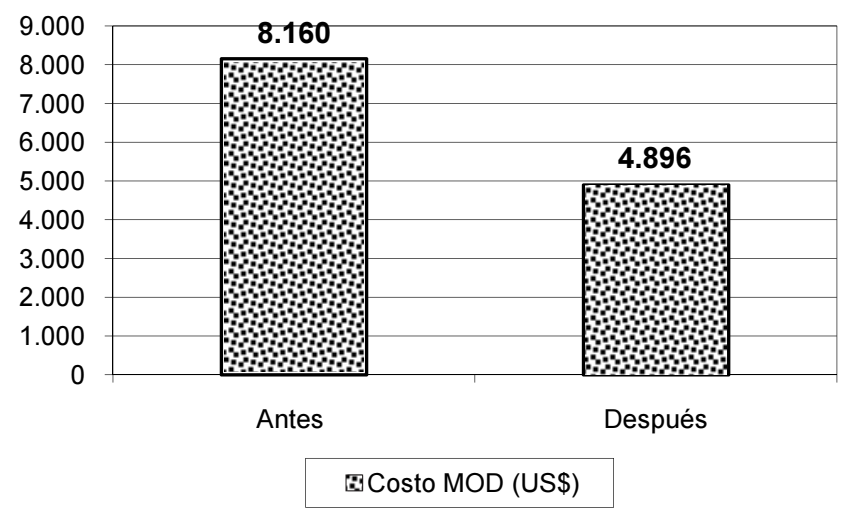

Fuente: Facusa. Estadísticas de la empresa.

capacidad de respuesta, así como operarios más capacitados, motivados, especializados y calificados que contribuyen en mayor medida a agregar valor a la organización.

\section{3}

Implementa su programa de mejora SMED (Single Minute Exchange of Die), cuya traducción al español es 'cambio de útiles en menos de 10 minutos', logrando reducir los tiempos de preparación de máquina durante los procesos de cuello de botella en alrededor del 20\%. Esto permitió a la empresa disminuir el tamaño de los lotes de producción, reducir los tiempos de fabricación, bajar los niveles de inventario, incrementar su productividad y lograr una mayor flexibilidad para adaptar su producción a las variaciones de la demanda. Asimismo, la incorporación de esta herramienta contribuyó a la identificación de problemas de calidad más rápidamente, reduciendo de esta forma los costos de no calidad relacionados con el reproceso, reinspecciones, desechos, producto no conforme, horas extras para subsanar errores y pérdida de tiempo. 

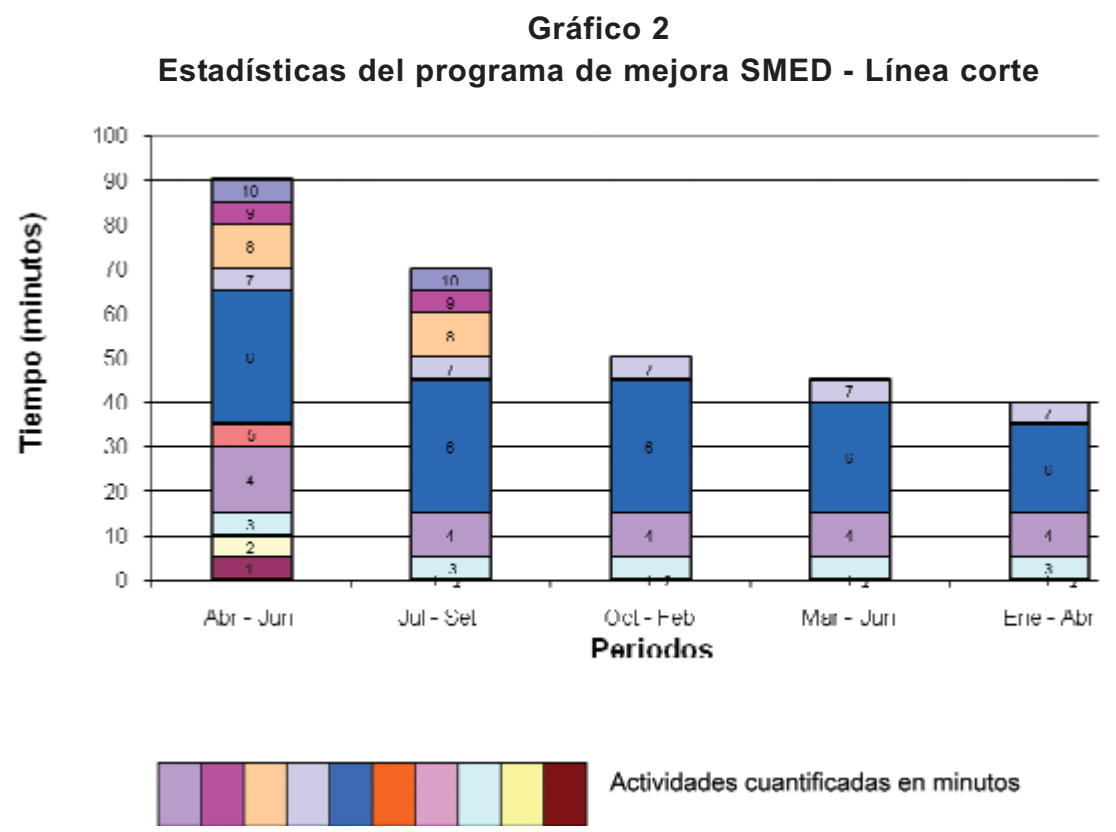

Fuente: Facusa. Estadísticas de la empresa.

2004

Facusa certifica su sistema de gestión de la calidad basado en la norma ISO9001:2000, para su proceso de diseño, desarrollo y fabricación de cubiertos y utensilios de acero inoxidable, concretando así uno de sus objetivos estratégicos mediante el cual buscaba introducir en la organización un modelo de aseguramiento de la calidad de sus productos, mediante un enfoque de trabajo basado en el cliente, que le permitiera a la empresa desarrollar y mantener de una manera sistemática su competitividad en el tiempo. En relación con el impacto de esta implementación se observó lo siguiente: mejoraron la motivación de los trabajadores y la coordinación entre las áreas, sus colaboradores desarrollaron un mayor grado de compromiso y sentido de pertenencia, se optimizó el uso de los recursos y se perfeccionó el control de los procesos. Finalmente, podemos concluir que la implementación de un sistema de gestión de la calidad le otorgó a Facusa una mayor capacidad de adaptación a las necesidades de los clientes tanto externos como internos, mediante el más óptimo control de las actividades operativas de la empresa. 


\section{Gráfico 3}

Estructura del sistema de calidad

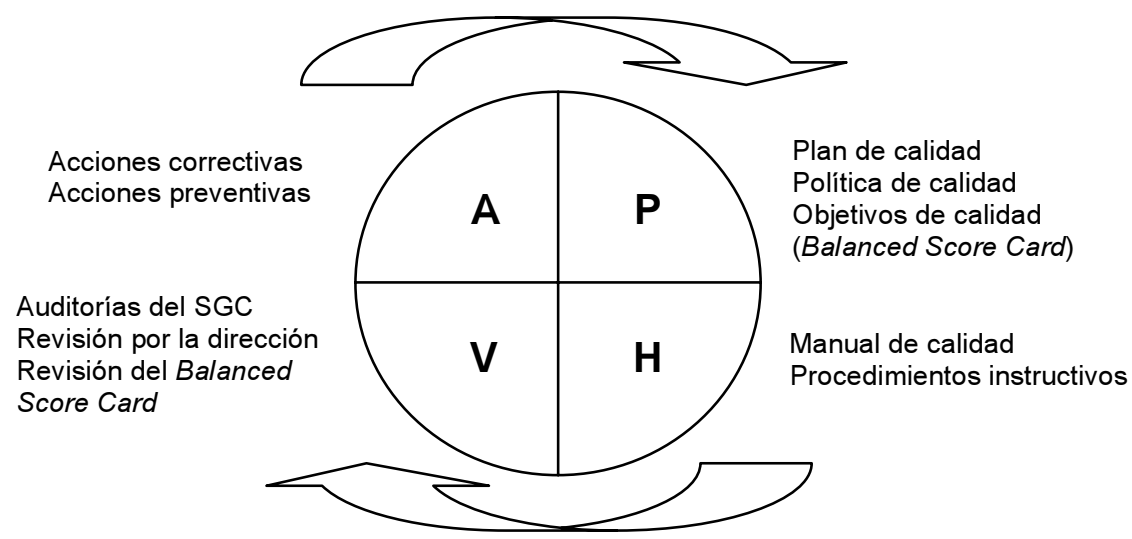

Fuente: Facusa. SGI de la empresa.

2005

Empieza a exportar a los países de la región, principalmente a los mercados colombiano y ecuatoriano. Durante este año la empresa exporta el $5 \%$ de su producción anual, incrementándose año tras año el volumen exportado. El 2008 exporta el 14\% de su producción anual, y alcanza un $19 \%$ durante el 2009 , con exportaciones al mercado boliviano. El objetivo estratégico en relación con las exportaciones es lograr que cada año los ingresos de la compañía dependan menos del mercado local y estar mejor preparados ante una contracción de la demanda interna; por ello, la meta en el corto plazo es exportar el $30 \%$ de la producción anual. A partir del 2010 se atiende el mercado colombiano a nivel nacional, para esto Facusa ha desarrollado una alianza estratégica con uno de los principales distribuidores mayoristas de este país. En relación con la penetración a nuevos mercados en el ámbito regional como estrategia de crecimiento se considera pertinente señalar que la empresa ha registrado de manera sostenida un crecimiento anual promedio del $15 \%$ de sus ventas en los últimos cinco años. Los principales destinos de sus exportaciones son Colombia, Ecuador, Bolivia y Venezuela. 


\section{Gráfico 4}

Evolución de las ventas 2005-2009

(en US\$)

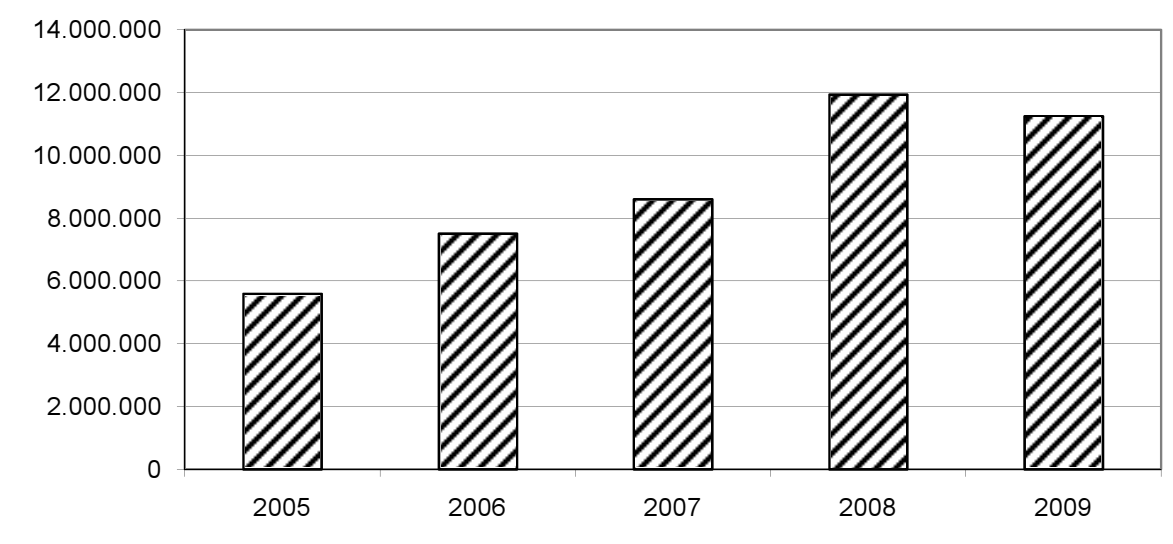

Fuente: Facusa.

2006

Implementa su tablero de control por medio del cual logra transformar sus objetivos y estrategias en indicadores tangibles. Mediante su cuadro de mando integral Facusa efectúa el seguimiento de los resultados financieros y operacionales y del mercado, midiendo la satisfacción del cliente a través de una encuesta, los resultados del proceso calculando el nivel de calidad y productividad global, y finalmente el desempeño y desarrollo del recurso humano evaluando el resultado de los programas de capacitación en la mejora de calidad y la eficiencia en el uso de los recursos.

La alta dirección de la empresa asume el cuadro de mando integral como una herramienta de gestión estratégica, tal como lo mencionan Robert Kaplan y David Norton: "El Cuadro de Mando Integral es más que un sistema de medición táctico u operativo" (2001: 23). 


\section{Gráfico 5 \\ Tablero de control-Facusa}

\section{Vista global de todos los procesos}

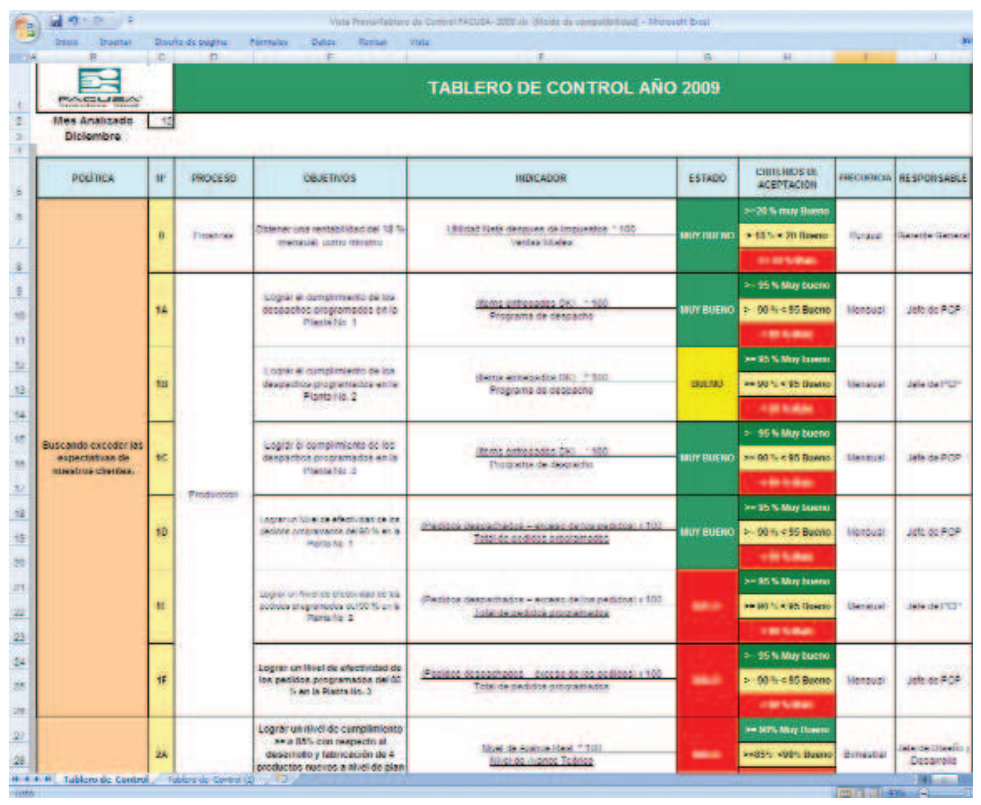

\section{Vista detallada de cada proceso}

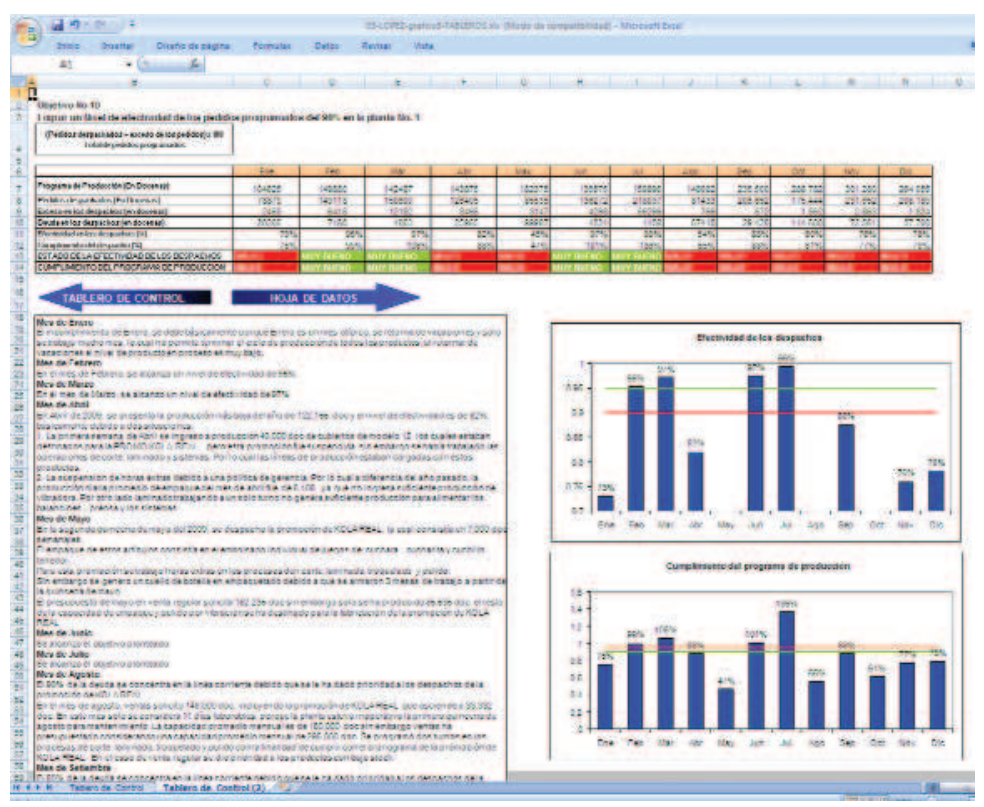

Leyenda BSC

Nivel de cumplimiento

$\square$ Muy bueno

$\square$ Bueno

$\square$ Malo 
Facusa se plantea como meta generar un mayor compromiso por parte de sus colaboradores, mediante el empoderamiento de cada proceso evidenciado en la aplicación del mantenimiento autónomo y el concepto de calidad en la fuente, cambio que se aplicó luego de un programa intensivo de capacitación que estuvo a cargo de los jefes de mantenimiento y calidad, respectivamente, y que fue dirigido a todo su personal operario y equipo de supervisores. La alta dirección pudo constatar, durante los sucesivos procesos de auditoría, un impacto positivo en el fortalecimiento y la madurez del sistema de gestión de la calidad luego de la introducción de este cambio en la cultura de trabajo. Asimismo, se pudo demostrar una mejora en la comunicación en todos los sentidos y un mayor grado de compromiso.

\section{8}

Facusa recertifica su Sistema de Gestión de la Calidad bajo la norma ISO9001:2008 y certifica su Sistema Integrado de Gestión basado en las normas ISO14001:2004 y OHSAS18001:2007, evidenciando de esta manera su compromiso con la seguridad y la salud de sus colaboradores y su anhelo como empresa de contribuir a la protección del medio ambiente. Se comprometió a desarrollar desde este año una cultura preventiva, planteándose como objetivo reducir a cero la ocurrencia de accidentes y mitigar todo impacto medioambiental que pudiera generarse a partir de su proceso de producción. En consecuencia, tiene definido un conjunto de procedimientos operacionales que ha difundido entre todo su personal y cuya finalidad es desarrollar en sus operaciones un desempeño seguro de trabajo y una cultura preventiva que identifique y elimine las condiciones laborales inseguras, mitigando y gestionando adecuadamente cualquier impacto medioambiental. 


\section{Gráfico 6}

ISO 14001:2004

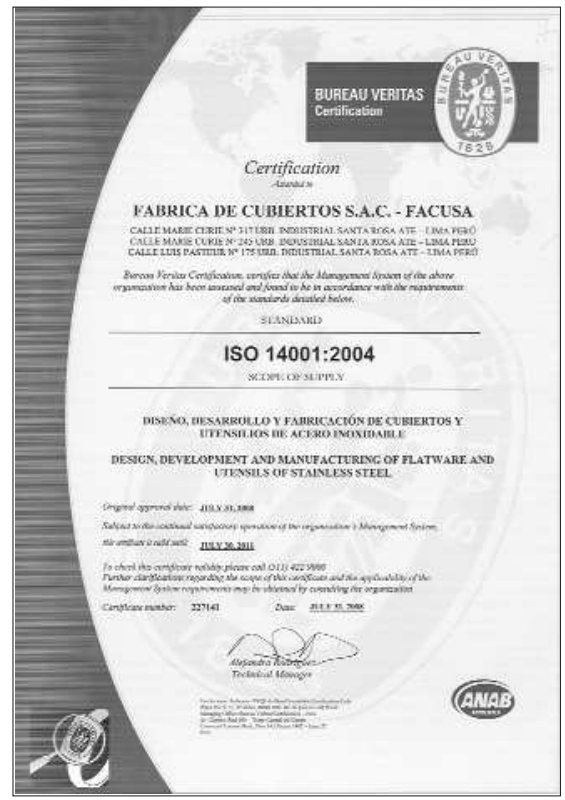

OHSAS 18001:2007

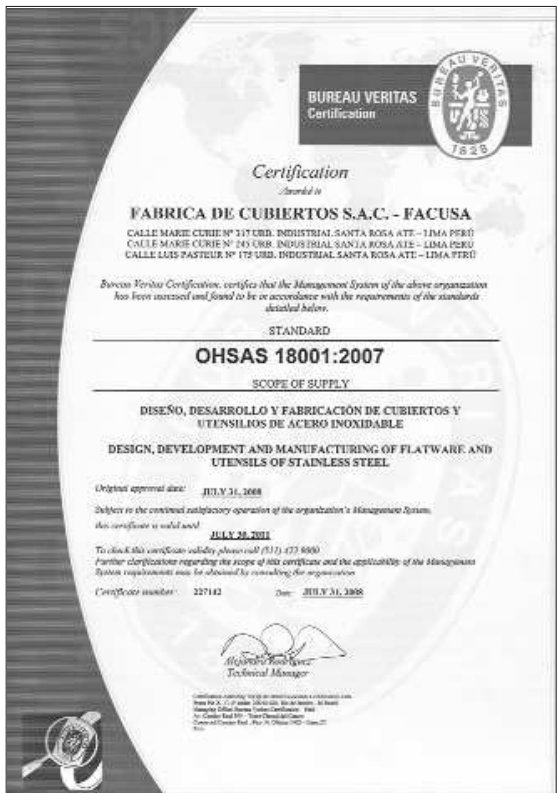

ISO 9001: 2008

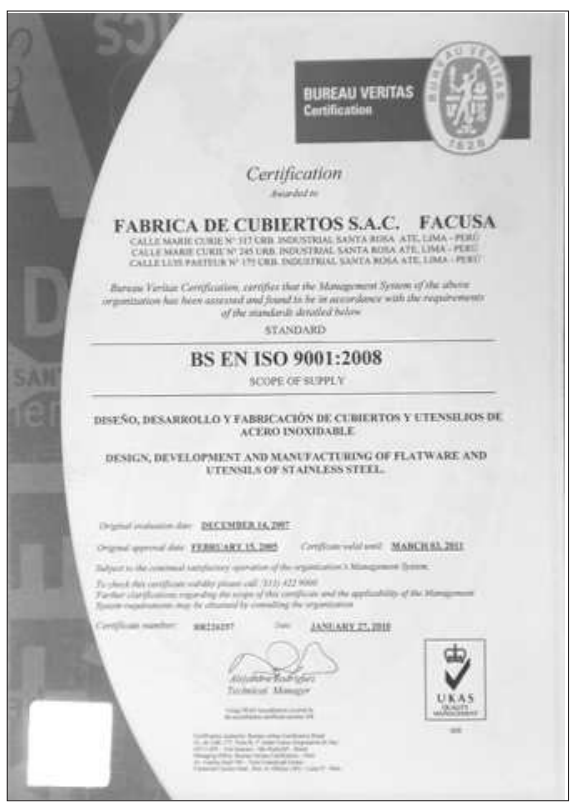

Fuente: Facusa. 
Durante el segundo semestre del 2009 inicia el diseño, desarrollo e implementación de su sistema ERP, con el objetivo de incrementar la eficiencia en sus operaciones. Para ello la alta dirección de la empresa conforma un equipo y nombra a un líder del proyecto, que ha tenido la responsabilidad de trabajar directamente en coordinación con la empresa responsable del desarrollo de su sistema de planeación de recursos empresariales ERP. Esta implantación le ha permitido a Facusa identificar oportunidades de mejora de sus procesos actuales de trabajo, con resultados favorables en la organización de los puestos de trabajo y aumentando en forma casi inmediata la productividad de algunos procesos, como la toma de inventarios, cuyo tiempo de ejecución se redujo en aproximadamente 50\%. Asimismo, se ha logrado integrar y utilizar la información a través de toda la organización en áreas claves como producción, compras, almacenes, finanzas, contabilidad, recursos humanos, logística y distribución, y finalmente márketing y ventas.

Uno de los principales objetivos que persigue la alta dirección de Facusa con la implementación de su sistema ERP ha sido aumentar su competitividad, controlar mejor sus operaciones e integrar su información. En relación con este último punto cabe mencionar que durante los últimos 12 años la compañía había tenido un manejo aislado de la información generada en los distintos procesos, condición que no permitía una integración y organización de la data generada en la organización, que apoyara la toma rápida y efectiva de decisiones en los niveles estratégico, táctico y operativo, y en procesos tan críticos como finanzas, distribución y manufactura. Otras de las ventajas adicionales que se están evidenciando en la empresa a partir de la implementación de esta solución, y que se encuentran aún en proceso de validación, son: una mayor confiabilidad de la información, reducción de los tiempos de atención de reclamos, mejora en los tiempos de respuesta, reducción de costos administrativos y reducción de los inventarios.

\section{CRECIMIENTO SOSTENIDO}

Es importante mencionar que Facusa aumentó sus ventas de manera sostenida en los últimos cinco años, con un incremento anual promedio del $15 \%$, pasando de facturar en alrededor de 5.000 .000 en el 2005 a alrededor de 10.000 .000 en el 2009. Durante los años 2008 y 2009 logró consolidar su presencia en los mercados de Colombia y Ecuador, 
y localmente mantiene una participación en el mercado peruano de cubiertos y utensilios de acero inoxidable de alrededor del $60 \%$. La empresa ha incrementado su producción anual de manera constante en la última década, pasando de producir alrededor de 960.000 docenas en el año 2000 a alrededor de 2.500 .000 en el 2008, a una tasa de crecimiento anual que fluctuó entre $12 \%$ y $15 \%$; teniendo como meta para el mediano plazo llegar a producir 6 millones de docenas de cubiertos y utensilios al año.

\section{Gráfico 7}

Evolución de la producción 2000-2009

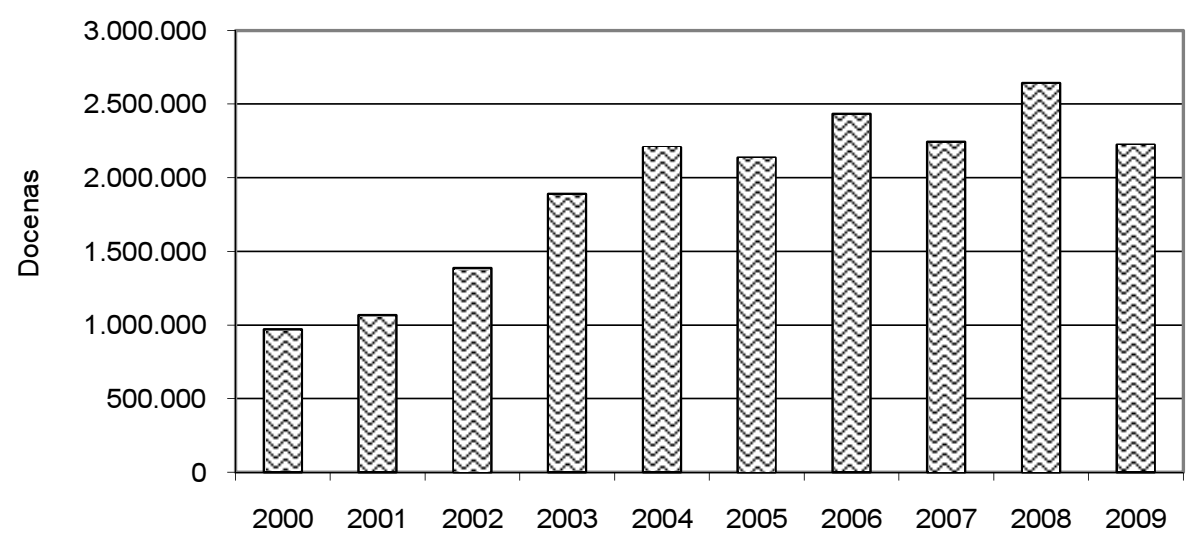

Fuente: Facusa. Estadísticas de la empresa.

Gráfico 8

Evolución de las ventas 2005-2009 (en US\$)

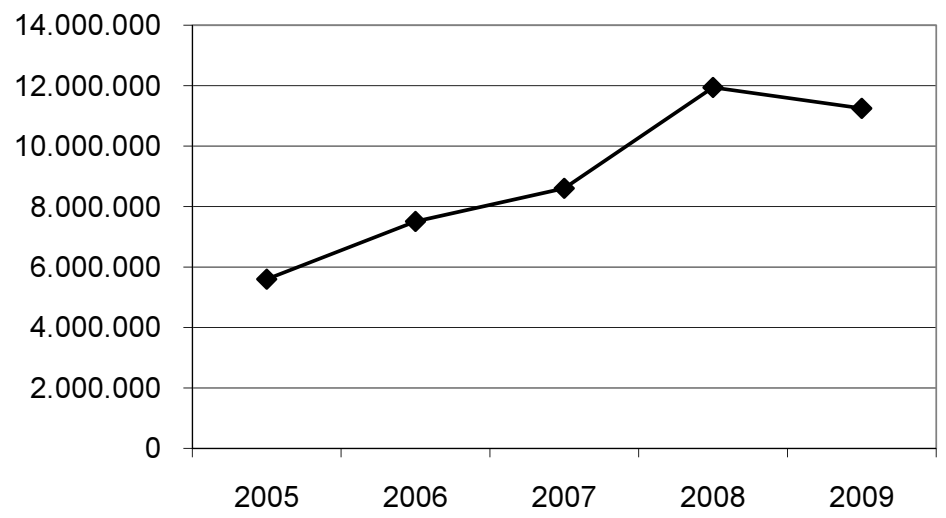

Fuente: Facusa. Estadísticas de la empresa. 


\section{POSICIONAMIENTO DE LA EMPRESA}

Actualmente, Facusa está posicionada como una marca líder en la fabricación y comercialización de cubiertos y utensilios de acero inoxidable de alta calidad y a un precio competitivo. Uno de los retos que enfrenta la empresa en el mercado globalizado es la cada vez más fuerte competencia de los productos importados procedentes principalmente de China e India.

\section{INNOVACIÓN TECNOLÓGICA}

Desde hace una década, Facusa tiene como política de empresa la reinversión del $30 \%$ de sus utilidades en la innovación y mejora tecnológica de sus productos y procesos mediante la automatización de estos y la renovación de su maquinaria, logrando incrementos de productividad de la mano de obra en los cuellos de botella de hasta $400 \%$. Adicionalmente, le ha permitido integrarse verticalmente hacia atrás, posibilitando la fabricación de sus propias herramientas, matrices, moldes y componentes, que luego utiliza en la fabricación de sus diversos productos. La flexibilidad que le otorga esta integración y la modernización constante de sus procesos de apoyo, como los talleres de máquinas herramientas, le ha permitido a la empresa elevar su nivel de innovación en los últimos cinco años, incrementando el número de nuevos productos lanzados al mercado por año, pasando de 3 en el 2005 a 7 en el 2009 .

\section{ENTORNO FUTURO}

Finalmente, la empresa proyecta para los próximos años una competencia más dura luego de la firma del tratado de libre comercio con China, que elimina prácticamente cualquier protección de tipo arancelaria para nuestra industria, para lo cual ha planteado, como parte de su estrategia empresarial para el mediano plazo, concentrar sus fuerzas en incrementar, desarrollar y consolidar su presencia en el mercado sudamericano, manteniendo su política de cero endeudamiento financiero e incrementando el porcentaje de su utilidad destinada a la reinversión del 30 al 50\%, para ser destinada fundamentalmente a la modernización de sus procesos mediante la adquisición de nueva maquinaria y equipos, y al aumento del ritmo de innovación de sus productos. 


\section{BIBLIOGRAFÍA}

Blanchard, Ken; Carlos, John P. y Alan Randolph (1996). Empowerment. Bogotá: Norma.

Chase, Richard B. y Nicholas Aquilano (1998). Dirección y administración de la producción y de las operaciones. México D. F.: Doyma Mexicana.

Domínguez, José Antonio; Álvarez Gil, María José; García, Santiago; Domínguez, Miguel Ángel y Antonio Ruiz Jiménez (1995). Dirección de operaciones. Aspectos tácticos y operativos en la producción y los servicios. Madrid: McGraw-Hill / Interamericana de España.

Goldratt, Eliyahu M. y Jeff Cox (1995). La meta. México D. F.: Ediciones Castillo.

Kaplan, Robert y David Norton (2001). El cuadro de mando integral. The Balanced Scorecard. Cambridge (Massachusetts): Harvard Business School Press.

Shank, John y Vijay Govindarajan (1995). Gerencia estratégica de costos. Bogotá: Norma.

Shingo, Shigeo (1991). Producción sin stocks: El sistema Shingo para la mejora continua. Madrid: Tecnologías de Gerencia y Producción.

Villajuana, Carlos (2006). Gestión estratégica integral. ¿Cómo ganar la guerra de intangibles? Lima: Villajuana Consultores.

Wheat, Barbara; Mills, Chuck y Mike Carnell (2004). Seis sigma. Una parábola sobre el camino hacia la excelencia y una "empresa esbelta”. Bogotá: Norma. 